\title{
RECENT RESULTS ON CHEMICAL MODIFICATION OF POLYMERS
}

\author{
PAUL REMPP \\ Centre de Recherches sur les Macromolécules, (CNRS), 6, rue Boussingault-67083 Strasbourg, Cedex, France
}

\begin{abstract}
Some characteristic features of chemical transformations of polymers are examined on three examples:
1. The reactions of various organo-lithium bases onto the ester carbonyls of polymethylmethacrylate are typical examples of one-step substitutions involving retardation, i.e. decreased reactivity of units vicinal to substituted ones.

2. The reaction of phenyl-lithium onto the carbonyl groups of PMMA is a typical two-step substitution process, with additional possibilities of cyclization via lactonization or via acetalization. To understand the reactions involved it is necessary to make use of model-molecules and of copolymers where cyclizations are avoided. It was established that retardation characterizes the first step (ketone formation) but the probability of the second step to occur subsequently is rather high. Lactonization (by attack of an alcoolate onto a vicinal ester) is therefore somewhat privileged.

3. Chemical modification of polyvinylalcohol by metallation and subsequent reactions of alkylhalides or of propane sultone was investigated thoroughly. It was found that sequential substitution was obtained. This is due to enhanced reactivity of units vicinal to substituted ones (case or propane sultone); but it may also be due to the tendency of side chains to crystallize in double layers, in which case the placement of the substituted units is determined by physical effects rather than by purely chemical factors.
\end{abstract}

\section{INTRODUCTION}

Chemical transformations of high polymers have been of increasing interest for the past decade, although they have been used extensively for a long time for manifold purposes: chemical modification of cellulose has been a wide field of investigation, and vinyl polymers have also been subject to chemical reactions: hydrolysis of polyvinylacetate to polyvinylalcohol, sulfonation of polystyrene, chlorination of polyethylene have been applied industrially. The modification is of special interest when the desired polymer cannot be obtained directly from its monomer, or when a copolymer of desired composition is wanted, which cannot be obtained easily by radical copolymerization of the two corresponding monomers, owing to the reactivity ratios of the system, or to the inability of a monomer to polymerize.

The investigation of a chemical reaction applied to the pendant functions of polymer species requires much care and very adequate characterization of the modified polymers. There may be side reactions involved, leading to chain scission (or in some cases to crosslinking). Other side reactions may lead to unwanted functions which can remain attached to the chain. As an example, the sulfonation of polystyrene by $\mathrm{H}_{2} \mathrm{SO}_{4}$ is accompanied by oxidative chain scissions and by formation of sulfone bridges.

Even if no unwanted reactions occur on the polymer chain, the process of chemical transformation of polymers is in itself quite complicated. The reactivity of a given site on the polymer chain depends upon whether the neighboring sites have already reacted or not. If we consider triads of monomer units, ${ }^{1-4}$ three rate constants can be defined for the reaction of function $A$ to yield a

group $B$ : They are generally called $k_{0}$ for $A \stackrel{*}{A} A$ triads, $k_{1}$ *

for $A A B$ (or $B A A$ ) triads and $k_{2}$ for $B A B$ triads, the asterisk indicating the unit which is supposed to react.

In only very few cases no appreciable influence is exerted on the reactivity of a $A^{*}$ function by the neighboring units. ${ }^{5}$ In such a case the chemical transformation retains a random character up to very high conversion, and for any fractional conversion the distribution of $A$ and $B$ units along the chain is strictly Bernouillian. Incidently such a random distribution of monomer units-governed by one single probability factor-is attained in random copolymerization only if the system is ideal, i.e. $r_{a} \cdot r_{b}=1$.

In numerous other cases ${ }^{6-9}$ the reactivity of a $A$ function to yield a $B$ function is decreased when the neighboring units have already reacted. This may be due to interactions, or to repelling charges (in the case of reactions involving ions) or even to steric factors. In such cases, characterized by:

$$
k_{0}>k_{1} \geqslant k_{2}
$$

it is not possible to attain complete conversion of the $A$ to $B$ units, and the distribution of substituted units along the chain is obviously Markovian. In some cases alternation is privileged, in other cases, when $k_{1}$ is still greater than $k_{2}$, the formation of $A B B$ triads is favored and if $k_{2}=0$ the maximum possible yield does not exceed $2 / 3$. In theses systems the fluctuations in composition remain quite low.

There are also some cases in which the reactivity of an $A$ function is enhanced by the presence of neighboring substituted units. ${ }^{10-12}$ This is due usually either to activation phenomena (as the allylic activation in the dehydrochlorination process of $\mathrm{PVC}^{11}$ ) or by an intermediate cyclization (as in the alkaline hydrolysis of isotactic PMMA $^{12}$ ). In this case $k_{0}<k_{1} \leqslant k_{2}$ and the result of the reaction is sequential substitution. In some cases, high yields of transformation can be attained. When fractional conversion are aimed fluctuations in composition may become important within the sample, ${ }^{4,13}$ showing that different molecules of the sample may have undergone the reaction to quite different extents. As to the position of substituents along the chain is concerned, ${ }^{14,15}$ the $B B B$ triads are obviously favored with respect to $B A B$, and even $B A A$ triads.

Another important factor to consider is the possibility 
of multiple reactions onto a function along the backbone. There are several kinds of multiple reactions the most important of which are:

Successive transformations of a given function A linked to the polymer chain, by attack of two (or even more) molecules of reagent; the obtained macromolecule is then a terpolymer, constituted of the remaining $\mathrm{A}$ (unsubstituted) units, of B units, reacted once, and of C units, twice reacted. The investigation of such a system is very complicated because of the competition between $\mathrm{A}$ and $B$ units, both able to react with the same reagent: $A \rightarrow B$ and $B \rightarrow C$. Furthermore the influence of $B$ and $C$ units on the reactivity of neighboring, yet unreacted, $A$ units may be quite different and there are theoretically 6 types of triads to be considered, corresponding to 6 possibilities of rate constants for the A to B transformation, and just as many for the $\mathrm{B}$ to $\mathrm{C}$ transformation.

\begin{tabular}{|c|c|c|}
\hline 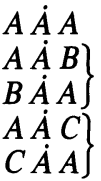 & $\begin{array}{l}C \dot{A} B \\
B \dot{A} C \\
B \dot{A} B \\
C \dot{A} C\end{array}$ & 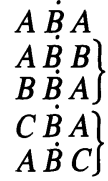 \\
\hline
\end{tabular}

Cyclisation may also occur as another possible type of multiple reactions. If it proceeds by attack of an unsubstituted A unit onto a neighboring sustituted B unit to form a cycle it is called intersequence cyclization., ${ }^{2,16}$ In some cases such a reaction can be used to measure the amount of $\mathrm{AB}$ diads present in the partly transformed polymer (or in a binary copolymer).

Another possible cyclization process involves reaction between two neighboring units of the same kind. ${ }^{2}$ This process is called intrasequence cyclization. It may occur between two initial units $A$, or between two already substituted B units. Many examples of inter- and of intrasequence cyclizations $\mathrm{s}^{2,16-20}$ have been described in recent years in literature. It should be noted that cyclizations never attain quantitative yields since in such random processes, there are always some units left over. The number of units left over in an intrasequence cyclization within a given sequence of $n$ units can be calculated using the wellknown recurrence formula. ${ }^{21}$

$$
\begin{aligned}
& \text {-Phenylthiomethyl- } \mathrm{Li}^{25,26} \\
& \text {-Methylsulfinylmethyl-Li (Dimsyl-Li) }{ }^{27} \\
& \text {-Methylsulfonylmethyl- } \mathrm{Li}^{27,28} \\
& \text {-Dimethylaminosulfonylmethyl- } \mathrm{Li}^{27} \\
& \text {-Methoxysulfonylmethyl- } \mathrm{Li}^{29} \\
& -\alpha \text {-picolyl-lithium }{ }^{30}
\end{aligned}
$$

$$
S_{n}=\frac{2}{n-1} \sum_{1}^{n-2} S_{i}=\frac{n-2}{n-1} S_{n-1}+\frac{2}{n-1} S_{n-2}
$$

The limiting conversion in the case of a homopolymer is therefore $1-1 / \mathrm{e}^{2}$, which is about $86.5 \%$.

In this presentation we shall illustrate these features of chemical modification of polymers by some results obtained over the past years at the Centre de Recherches sur les Macromolécules; two different polymers were used as substrates, namely polymethylmethacrylate and polyvinylalcohol, and very accurate characterization techniques were used to establish the characteristics of the reactions investigated. It will be seen that neighboring effects in polymer reactions can be due to many different factors.

\section{NUCLEOPHILIC SUBSTITUTION ONTO THE} ESTER FUNCTIONS OF POLYMETHYLMETHACRYLATE

Polymethylmethacrylate (PMMA) is a well defined linear polymer, soluble in many solvents, rather stable, and rather easy to characterize. Furthermore high contents of isotactic or of syndiotactic triads can be obtained by choosing the preparation conditions. PMMA is therefore an adequate polymer for investigations on chemical transformations of pendant ester functions. In fact the ester carbonyles are easily attacked by various nucleophiles such as hydroxides (hydrolysis ${ }^{2,1,22}$ ) amines (aminolysis ${ }^{23,24}$ ) etc. We focused our attention to reactions of various organolithium compounds onto the carbonyles of the ester functions of PMMA. This reaction may be either a one-step or a two-step process.<smiles>CCC(C)(CC)C(=O)OC</smiles>

(A)<smiles>[R]C(=O)C(C)(CC)CC[CH]C</smiles>

(B)
(C)
The reaction stops after the first step when the reactivity of the obtained ketonic groups is greatly decreased either by steric effects, or by resonance stabilization involving enolate anions. If the keto-enolic equilibrium is shifted very much back to the ketone, or if no such equilibrium is even possible (as in the case of phenone groups) one can expect $B$ to react with further nucleophile to yield tertiary alcohol functions (C).

\section{One step substitution on PMMA}

The investigated organolithium derivatives which, upon attack of the ester functions of PMMA, lead to ketone functions largely enolized in alkaline media, comprize the following compounds some of which have been already studied for micro-molecular esters by several authors:

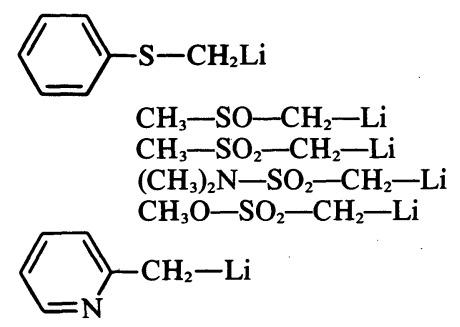

They lead respectively to keto- $\beta$-sulfide, keto- $\beta$ sulfoxide, keto- $\beta$-sulfone, keto- $\beta$-sulfonamide, keto- $\beta$ sulfonate and keto- $\beta$-pyridyl groups which are all ionizable, some of them even exhibiting the character of a strong organic acid.

Stoichiometry. The nucleophilic substitution of one ester function of the PMMA requires two molecules of the organolithium reagent: ${ }^{31}$ the first to attack the carbonyl, the second to deprotonate the central methylene group of the keto- $\beta$-sulfide, keto- $\beta$-sulfoxide, or keto- $\beta$ sulfone function. This methylene group is always more 
acidic than the precursor of the organometallics (DMSO, dimethylsulfone...) and in most cases it is also more acidic than methanol. However, owing to the difference in basicity between the reagent used and lithium methoxide, the latter compound-which is formed in the reaction process-is not contributing much to the deprotonation of the methylene group. A twofold excess of the organolithium compound is therefore required.

Side reactions. No side reactions can be detected when the reaction between the organolithium and PMMA is carried out at room temperature in DMSO solution, or in mixtures of HMPA and THF. The degree of polymerization of the backbone stays almost constant, and no other function but the one expected and the initial ester group could be detected.

Reactions carried out at higher temperature $\left(60^{\circ} \mathrm{C}\right)$ give rise to very few chain scissions.

From experiments on dimeric model compounds, such as dimethylglutarate and dimethyl-cyclohexane dicarboxylate-as well as from literature data ${ }^{28}$-it was expected that some cyclization may take place, mainly according to the following scheme:<smiles>COC(=O)C(C)(C)CC(C)(C)C</smiles><smiles>CCCC</smiles>

This intrasequence type cyclization involves attack of an already substituted B unit by the anion of a vicinal unit of the same type. But very carefull spectroscopic determinations on PMMA modified to various extents, as well as NMR measurements failed to identify these cycles, and it can therefore be assumed that cyclization occurs to less than $1 \%$, even if the reaction is carried out at elevated temperature and in the presence of a large excess of nucleophile.

Reaction kinetics. The reaction yields stay usually far below unity at $25^{\circ} \mathrm{C}$ and in most cases the maximum degree of substitution (DS) reaches up to $60 \%$. This result indicates the existence of a retardation effect. The rate constants $k_{0}, k_{1}, k_{2}$ for the substitution of the central unit in $A \dot{A} A, A \dot{A} B$ and $B \dot{A} B$ triads, respectively, decrease in that order. If the DS is limited to less than $2 / 3$ one can assume $k_{2}$ to be very close to zero.'

Kinetic investigations of the reaction of dimsyl anions and of dimethylsulfonyl anions onto PMMA were carried out at $25^{\circ} \mathrm{C}$; the amount of methanol formed was measured by vapor phase chromatography and the degree of substitution was determined by elemental analysis. From the plots of the DS vs time (Fig. 1) the apparent rate constant of the reaction can be calculated at any time. Using Fuoss's method ${ }^{6}$ to give account of the variation of $k_{\text {app }}$ with time, it was found that two rate constants, are sufficient to fit satisfactorily the experimental curve (Figs. 2,3 ) and it is thus confirmed that $k_{2}$ is very close to zero, if the reaction is carried out at $25^{\circ} \mathrm{C}$.

The following kinetic results have been obtained:

-for the reaction of $\mathrm{CH}_{3} \mathrm{SO}_{2} \mathrm{CH}_{2} \mathrm{Li}$ onto syndiotactic PMMA, at $25^{\circ} \mathrm{C}$ in 5:1 DMSO-benzene mixture:

limiting conversion 52\%; (PMMA) $=0.133 \mathrm{~mol} / \mathrm{l}$.

$$
\begin{gathered}
k_{0}=9.9 \times 10^{-2} \quad k_{1}=5.5 \times 10^{-3} \quad k_{2} \simeq 0 \\
k_{0} / k_{1}=18\left[k \text { in } 1 \mathrm{~mol}^{-1} \mathrm{~min}^{-1}\right]
\end{gathered}
$$

-for the reaction of $\mathrm{CH}_{3} \mathrm{SO}_{2} \mathrm{CH}_{2} \mathrm{Li}$ onto isotactic PMMA at $25^{\circ} \mathrm{C}$ in the same solvent mixture:

limiting conversion $59 \%$

$$
\begin{gathered}
k_{0}=4.7 \times 10^{-1} \quad k_{1}=4.7 \times 10^{-1} \quad k_{2} \simeq 1.4 \times 10^{-3} \\
\frac{k_{0}}{k_{1}}=1 \quad \frac{k_{1}}{k_{2}}=300
\end{gathered}
$$

-for the reaction of $\mathrm{CH}_{3} \mathrm{SOCH}_{2} \mathrm{Na}$ onto "atactic" PMMA (70\% syndio.) in DMSO solution at $25^{\circ} \mathrm{C}$ :

$$
\begin{gathered}
k_{0}=8.33 \quad k_{1}=0.34 \quad k_{2} \simeq 0 \\
{\left[k \text { in } 1 \mathrm{~mol}^{-1} \mathrm{~min}^{-1}\right] .}
\end{gathered}
$$

The influence of the tacticity of PMMA on the substitution by the organolithium compounds is quite noticeable from the above results. Furthermore the highest degree of substitution obtained with dimethylamino-

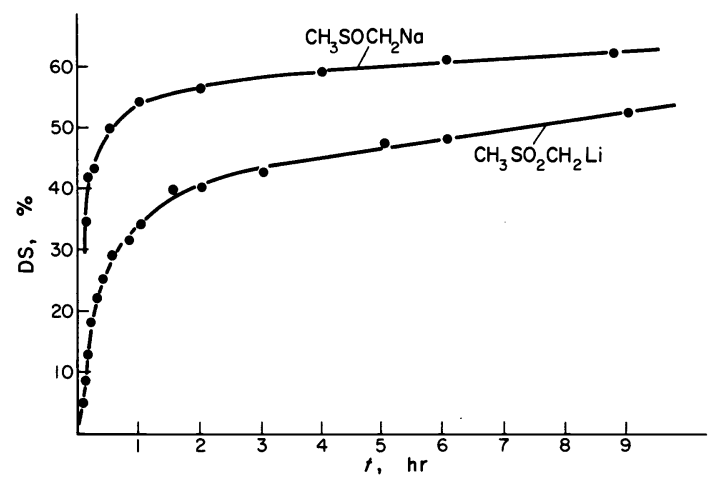

Fig. 1. Example of kinetic behavior of the substitution of PMMA by dimethylsulfinyl-Li, and by dimethyl-sulfonyl-Li. Degree of substitution (\%) vs time (hr).

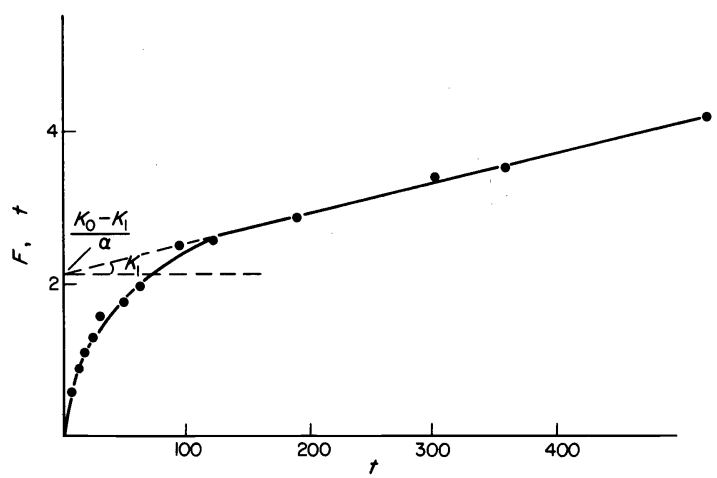

Fig. 2. Plot of $F(t)$ vs time $t$ to analyse the kinetic data according to Fuoss. 


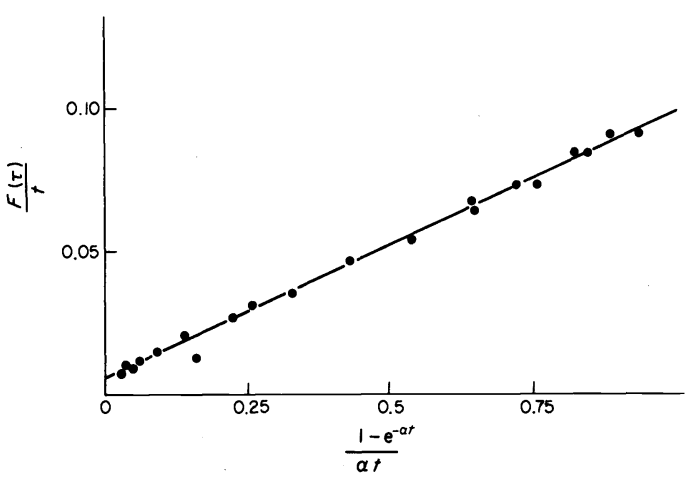

Fig. 3. Fuoss linearization plot: $[(1 / t) F(\tau)]$ vs $\left[\left(1-\mathrm{e}^{-\alpha t}\right) / \alpha t\right]$ indicating that two rate constants are sufficient to describe the phenomena involved.

sulfonylmethyl-Li, at $60^{\circ} \mathrm{C}$ is $75 \%$ in the case of syndiotactic PMMA, but it reaches $95 \%$ for isotactic PMMA. This shows that in syndiotactic PMMA the placement of the substituents leaves less space for the attack by a nucleophile than in the case of isotatic PMMA. Similar results have been obtained for the alkaline hydrolysis of PMMA and of isoprene-PMMA random copolymers. $^{32}$

Compositional heterogeneity. It has already been mentioned that when functions surrounded by already substituted groups exhibit a lower reactivity towards the reagent, the fluctuations in composition should remain quite low. This statement was checked very accurately by using the "cross fractionation" technique on a PMMA in which $36.6 \mathrm{~mol} \%$ of the ester functions had been replaced by keto- $\beta$-sulfonamide groups.

Cross fractionation has been first developed by Limanovich and Shtern ${ }^{33}$ and was applied since then to many copolymer systems, like styrene-butadiene ${ }^{34}$ or styrene-MMA copolymers. ${ }^{35}$ The principle is to choose two different solvent-precipitant systems, both very sensitive to changes in composition, but in opposite directions. Figure 4 shows a plot of the $\gamma$ (volume fraction in the precipitant) vs the degree of substitution of PMMA, for several solvent-precipitant systems. The systems chosen were chloroform/diethylether, and DMF/water. Diethylether is more efficient as a precipitant as the DS increases, whereas for water it is the opposite. Once the systems have been chosen, a fractional precipitation is carried out using one of the systems, and each fraction recovered is submitted to a second fractionation using the other solvent-precipitant mixture. Thus one gets a series of fractions, the composition of which (i.e. the DS) is determined analytically. Figure 5 shows the obtained results. It can be seen that for the purpose of this

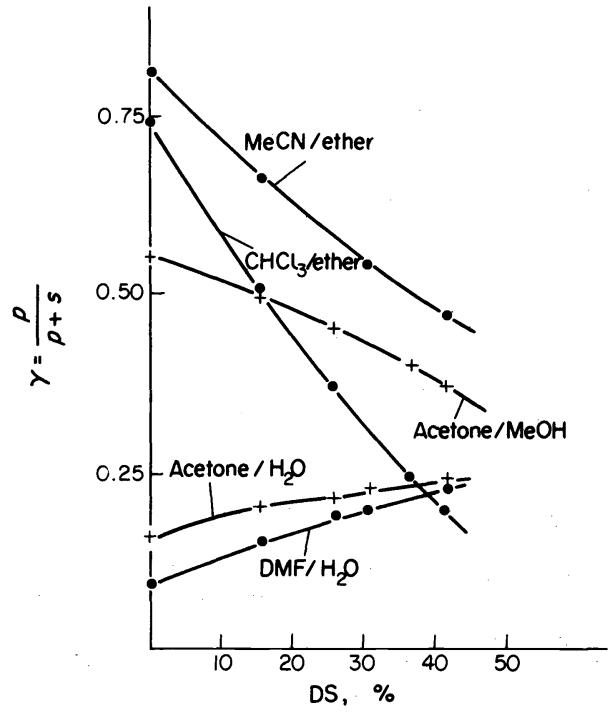

Fig. 4. Plot of the volume fraction $\gamma$ of precipitant in various solvent-precipitant systems as function of the degree of substitution of PMMA by dimethyl-aminosulfonylmethyl-Li.

investigation cross-fractionation is a most powerful tool. But it also shows that the fluctuations in composition within that sample are very small-as it was expected from the kinetic data-and the mean square deviation from the average composition, calculated over all fractions is of the order of $1.4 \times 10^{-2}$, which is even less than it was reported for an azeotropic styrene-MMA random copolymer. ${ }^{35}$

Further investigations of the compositional heterogeneity were carried out by the method first described by Bushuk and Benoit. ${ }^{36}$ Here lightscattering measurements were carried out on the whole modified polymer, in various solvents chosen to cover a wide range of refraction indices. ${ }^{37}$ In case of compositional heterogeneity the "apparent" molecular weights obtained in these various solvents differ noticeably; the values obtained are closest to the true molecular weight of the polymer when the refraction index increment is the largest. In the case of our partly modified PMMA the values of $M$ remain constant within experimental error for all solvents used, which confirms the satisfactory compositional homogeneity.

\section{Two-step substitution of PMMA}

It can be expected that several reaction paths can be taken upon attack of the ester function of PMMA by phenyl-lithium. These reactions are schematized below:<smiles>COC(=O)C(C)(C)CC(C)(C)C(=O)OC</smiles>

(A)<smiles>CC1(C)CC(C)(C)C([O-])(O)OC1([O-])[O-]</smiles><smiles>CC1CC1</smiles><smiles>COC(=O)CC(C)(C)C(=O)OC</smiles>

(B)<smiles>CCCC(C)(C)C(=O)[O-]</smiles><smiles>C[14CH3]</smiles><smiles></smiles><smiles>[2H]C1([18O])OC(=O)C(C)(C)CC1(C)C</smiles> 


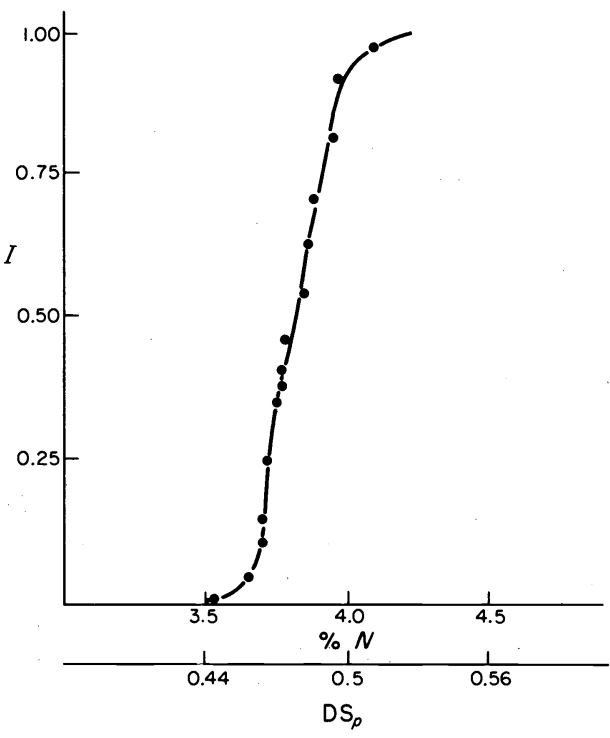

Fig. 5. Integral distribution of composition within a sample of partly substituted PMMA (by dimethylaminosulfonylmethyl-Li).

Average degree of substitution $48 \%$ by weight, or $32.6 \mathrm{~mol} \%$.

There are two products of substitution: ketone (methacrylophenone unit, (B), or tertiary alcohol (diphenylmethallylic alcohol unit, (C). Furthermore two types of cyclization can be expected: lactonization upon attack of an alcoholate (B) onto a vicinal ester (A); acetalization upon attack of an alcoholate $(C)$ onto a vicinal ketone (B) function; this reaction may progress further if the next unit along the chain is again a ketone $B$. One can therefore expect a partially substituted PMMA to exhibit 5 different types of units: ester, ketone, alcohol, lactone and acetal, and it can be easily imagined how complicated the problem becomes.

It was therefore interesting to characterize first the trend towards double substitution by measuring the ratio $r$ of the two rate constants $k_{a}$ and $k_{b}$. This was done on methylpivalate, in order to avoid complications arising from cyclizations. This case of two successive and competitive reactions can be treated according to Jungers ${ }^{38}$ if it is assumed that both steps are first order in the organometallics. Calling $x_{a}$ and $x_{b}$ the mole fractions of ester and of ketone, and $r=k_{a} / k_{b}$ one can use the following equation:

$$
x_{b}=\frac{r}{r-1}\left(x_{a}^{1 / r}-x_{a}\right)
$$

to relate the fraction of ketone functions to that of the remaining ester groups. If $r$ is smaller than unity the maximum value of $x_{b}$ is attained for $r=x_{a} / x_{b}$ (Fig. 6). These are some results, taken as examples:

$$
\text { in toluene }-70^{\circ} \mathrm{C} \quad x_{a}=0.70 \quad x_{b}=0.105 \quad x_{c}=0.195
$$$$
\text { in benzene }+20^{\circ} \mathrm{C} \quad x_{a}=0.95 \quad x_{b}=0.014, \quad x_{c}=0.036 \text {. }
$$

From these results, it follows that:

$$
\begin{aligned}
& r=0.14 \text { in toluene at }-70^{\circ} \mathrm{C} \\
& r=0.016 \text { in benzene at }+20^{\circ} \mathrm{C} .
\end{aligned}
$$

The second step of substitution is favored with respect to the first and therefore the amount of ketone functions present in the reaction mixture stays always low, whatever the conversion attained.

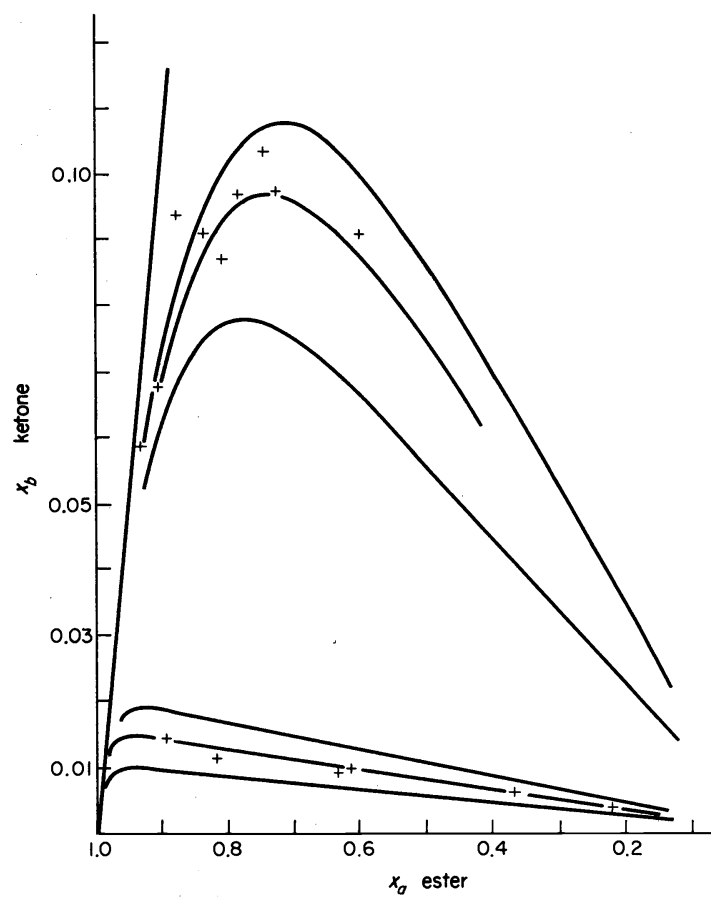

Fig. 6. Mole fraction of ketone $x_{b}$ vs mole fraction of remaining ester $x_{a}$. The curves are theoretical plots for given values of $r$. The experimental values obtained for methylpivalate are indicated by crosses $(+)$.

Another series of experiments was carried out on a styrene-methylmethacrylate azeotropic random copolymer, in which the percentage of isolated MMA units is rather high as it can be seen from the following table calculated using the wellknown reactivity ratios for that system:

number of units $n$ in MMA sequence fraction of sequences having $n$ MMA units

$$
\begin{array}{ccccc}
1 & 2 & 3 & 4 & \geqslant 5 \\
0.706 & 0.208 & 0.061 & 0.018 & 0.007
\end{array}
$$

The following table shows the results of substitution experiments on this random copolymer (48\% MMA) by phenyl-Li.

Table 1

\begin{tabular}{lllll}
\hline $\begin{array}{l}\text { DS } \\
(\%)\end{array}$ & \multicolumn{1}{c}{$x_{a}$} & \multicolumn{1}{c}{$x_{b}$} & \multicolumn{1}{c}{$x_{c}$} & $\begin{array}{c}x_{d} \\
\text { cyclized }\end{array}$ \\
\hline 13 & 0.87 & 0.097 & 0.023 & 0.01 \\
30 & 0.70 & 0.18 & 0.11 & 0.01 \\
56.5 & 0.435 & 0.24 & 0.27 & 0.05 \\
78 & 0.22 & 0.196 & 0.50 & 0.09 \\
96 & 0.04 & & 0.85 & 0.10 \\
\hline
\end{tabular}

$\mathrm{NB}: x_{a}$ is the fraction of remaining ester units in the copolymer.

From Table 1 it follows that the second step of reaction (addition of $\phi \mathrm{Li}$ onto the ketone functions) is far less favored in that copolymer than it is for a model-molecule. The $r$ ratio which fits best is of the order of 0.5 (Fig. 7). This result illustrates the higher steric hindrance around the ketone functions in the polymer which impedes somewhat further addition of $\phi \mathrm{Li}$, whereas 
pivalophenone reacts very readily with the same reagent.

A similar procedure was used to investigate the acetalization reaction. Here polymethacrylophenone was used as starting polymer, and the reaction of phenyl-Li on this polymer can only lead to tertiary alcohols and to acetalization (by reaction of an alcoholate onto a vicinal ketone). It was established that the number of cycles formed is far less than the number of possible cycles.

Obviously, among the two possible cyclization reactions involved in the overall reaction process of phenyllithium onto PMMA, acetalization occurs to a much lesser extent than lactonization.

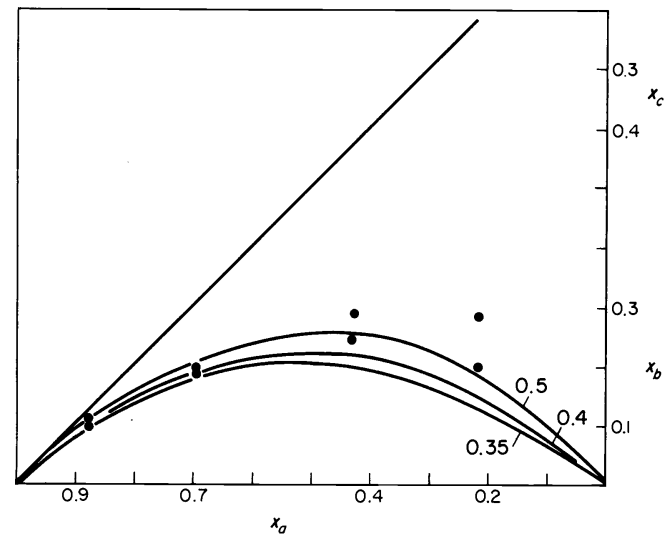

Fig. 7. Phenyl-Li substitution onto the ester functions of a styrene-MMA azeotropic copolymer. Composition plot $x_{b}$ vs $x_{a}$ for various values of the ratio $r=k_{a} / k_{b}$. The points refer to experimental results.

To try to systematically identify all functions present on a PMMA chain after it has been submitted to reaction with phenyl-lithium to a given degree of substitution the following characterization techniques have been used:

NMR which allows determination of the amount of remaining ester functions and of the total phenyl content of the polymer.

Infra-red spectroscopy which could be used to determine the amount of ester carbonyls and of ketone carbonyls.

Ultraviolet absorption to measure the phenone groups $(\phi-\mathrm{CO})$.

Chemical determination of the alcohol functions (Zerewetinov method) which yields the sum of tertiary alcohol and of hemiacetal functions, the acetals being not hydrolyzed under the conditions chosen.

Flash pyrolysis at $600^{\circ} \mathrm{C}$ in the absence of oxygen, a technique which takes advantage of the depolymerizability of backbone and allows the measurement of the amount of MMA and of methacrylophenone units in the polymer.

Systematic comparisons were made to check the self consistency and the accuracy of the experimental results. A plot of the experimental data is presented on Fig. 8.

From Fig. 8 it can be seen that:

The fraction of ketone functions never exceeds $20 \%$, whatever the degree of conversion attained.

The amount of detectable alcohol functions is of the same order of magnitude, except for very high conversions.

Around $50 \%$ of the units are involved in cycles, except for very low conversions.

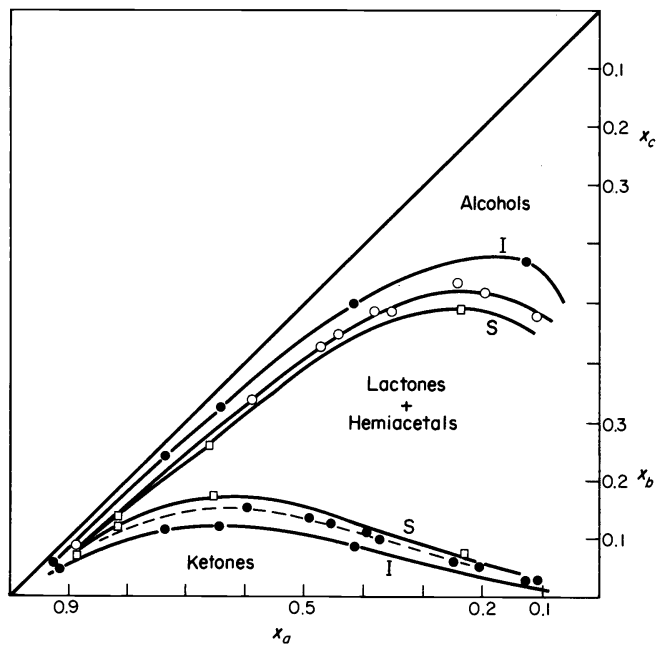

Fig. 8. Plot of the composition of PMMA partly substituted by phenyl-Li: $x_{a}$ is the fraction of remaining ester functions $x_{b}$ is the mole fraction of the ketone functions (lower curves) and $x_{c}$ is the mole fraction of the tertiary alcohols (higher curves, plotted downwards). The difference indicates the amount of cyclized units (lactones and hemiacetals). I and S refer to results obtained on isotactic and on syndiotactic PMMA.

The tacticity of the PMMA plays an important role, since isotactic PMMA yields less ketone and less alcohol upon reaction with phenyl-lithium, showing an enhanced tendency to cyclize with respect to syndiotactic triads.

From all these results it can be concluded that:

Retardation is the characteristic feature of this substitution, since for a given degree of substitution the proportion of $\mathrm{AB}$ (and $\mathrm{AC}$ ) diads is higher than predicted from Bernouillian statistics.

Whenever a B unit (ketone) has been formed the probability that it undergoes further reaction to tertiary alcoholate $\mathrm{C}$ ) is rather high. In other words $r$ is definitely lower than unity, though it is impossible here to determine it, even approximately.

The probability of cyclization by attack of an alcoholate function onto a vicinal ester is rather high, to yield lactone functions.

The amount of acetalization, by attack of an alcoholate onto a vicinal ketone function is not very high, because of the decreased probability of formation of $\mathrm{BC}$ diads (due to the retardation) and moreover because of the fact that this type of cyclization does not occur to high yields (as shown from experiments with polymethacrylophenone).

\section{CHEMICAL MODFICATION OF POLYVINYLALCOHOL}

Polyvinylalcohol originates from saponification of polyvinylacetate and it usually still contains some acetate functions attached to the chain. Metallation of the hydroxide functions was carried out using dimsyl-sodium as metalating agent, with the aim of performing two types of reactions on the metalated PVA, namely:

addition of propanesultone onto the alcoholate groups which results in the formation of sulfonate labeled units.

Williamson ether synthesis with long chain alkyl halogenides to fit some units of the chain with long, crystallizable substituents.

The reactions can be schematized as follows: 


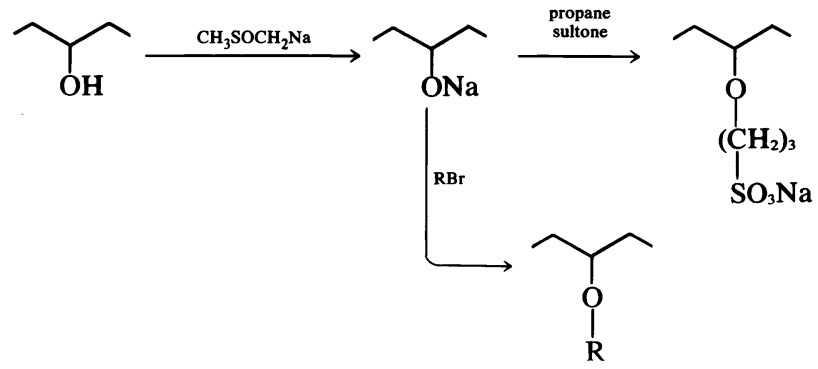

Metalation was performed in homogeneous medium (DMSO), at $60^{\circ} \mathrm{C}$ and degrees of substitution up to $70 \%$ were obtained within hours. ${ }^{39}$ This is not really a limiting degree of metalation but owing to the strong tendency of alcoholates to associate, even in polar solvents the reaction medium has become a gel, as $50 \%$ of the alcohol functions have been metalated.

No chain scissions occur upon metalation and no conclusion can be drawn from the kinetics as to what concerns the comparative triad reactivities. It should also be mentioned that remaining acetate functions are saponified during the process, as it can be seen from the i.r. spectra (the carbonyl absorption disappears).

\section{Sulfopropylation}

When treated with propane sultone, according to a method first described by Galin ${ }^{40}$ and Goethals, ${ }^{41}$ the acoholate of PVA yields sulfopropylated vinylether functions. The reaction is carried out in DMSO solution and it can be pushed to rather high yields. ${ }^{39}$ As shown on Fig. 9, the obtained DS is somewhat lower than the expected one, but up to $50 \%$ conversion the difference is not very large.

\section{Alkylation $^{42}$}

The reaction of alkylbromides onto the metalated PVA has been carried out in pure DMSO or in DMSO/THF mixtures, at $40^{\circ} \mathrm{C}$, and it proceeds to conversions never higher than $20 \%$. When long chain alkylbromides were

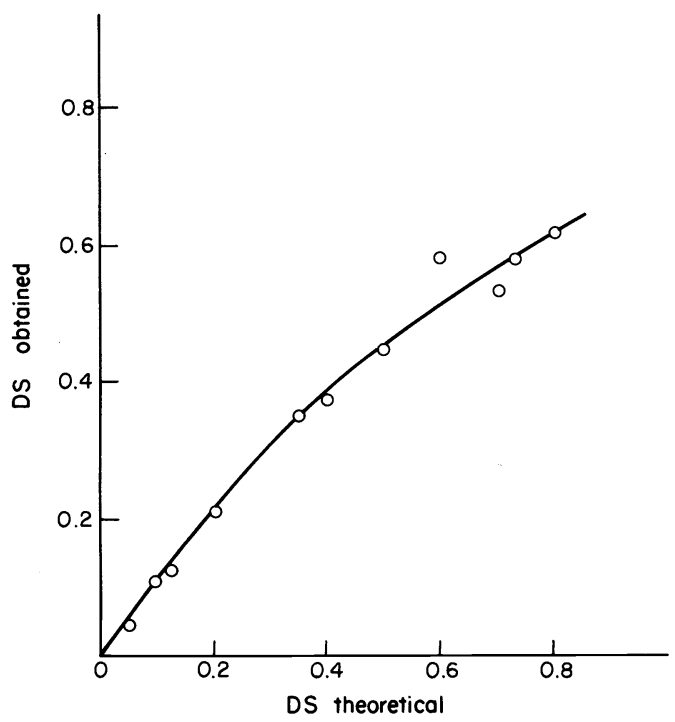

Fig. 9. Substitution of polyvinylalcohol by propanesultone: obtained substitution degree vs expected degree of substitution. used, the affinity of the grafted chains for DMSO is very low and phase separation occurs. The use of DMSO/THF mixtures is therefore preferable, but the yield of the Williamson reaction is not improved noticeably $\left(\mathrm{DS}_{\text {lim. }}\right.$ is 25 vs $22 \%$ in pure DMSO).

\section{Sulfopropylation and alkylation ${ }^{42}$}

Metalated PVA can be reacted successively with the two reagents, and one may get thus PVA chains grafted with sulfopropyl groups and with long parafinic chains. Propanesultone is always used first, to provide for satisfactory homogeneity of the reaction medium. The total number of grafted units never exceeds $65 \%$, but in no case have more than $22 \%$ of alkyl groups been linked to the backbone.

Distribution of the substituted units along the backbone At first sight the fractional limiting degree of substitution of $\mathrm{ONa}$ by $\mathrm{OR}$ or by $\mathrm{O}\left(\mathrm{CH}_{2}\right)_{3} \mathrm{SO}_{3} \mathrm{Na}$ units is an argument in favor of a retarding neighboring effect; in that case one would expect no fluctuations in composition within the sample, whatever the DS may be. However some properties of the PVA grafted twice, with hydrophilic and hydrophobic substituents seem to indicate that the placement of the units along the chain is not that expected in the case of a retardation effect. The ability of these polymers to yield good membranes, which swell appreciably in water, in spite of the long chain parafinic side chains which are hydrophobic would rather indicate sequential grafting.

To get more precise informations about the placement of each type of grafts, dehydration experiments were carried out using $\mathrm{KHSO}_{4}$ at $100^{\circ} \mathrm{C}$ in heptane. ${ }^{43}$ It is known that owing to the allylic activation this process is sequential and leads to sequences of double bonds. ${ }^{44}$ For a given degree of substitution, one can calculate, under the assumption of random grafting, the distribution of the unsubstituted sequences. A sequence of $w$ conjugated double bonds has a characteristic UV absorption and can thus be identified as such. Using a calibration process it is even possible to determine the fraction of sequences having 3,4 and up to 15 conjugated double bonds. This procedure will thus enable checking of the distribution of the substituted units: if it is random, there will be a satisfactory fit between the theoretical and the experimental curve. If there is retardation in the substitution reaction, the small sequences of double bonds will be privileged. If the substitution exhibits sequential characteristics the long sequences are favored.

Figure 10 shows the results obtained for PVA partly substituted by propanesultone, after dehydration of the remaining PVA units. Obviously the long sequences are favored very largely, which indicates that the sulfopropy- 


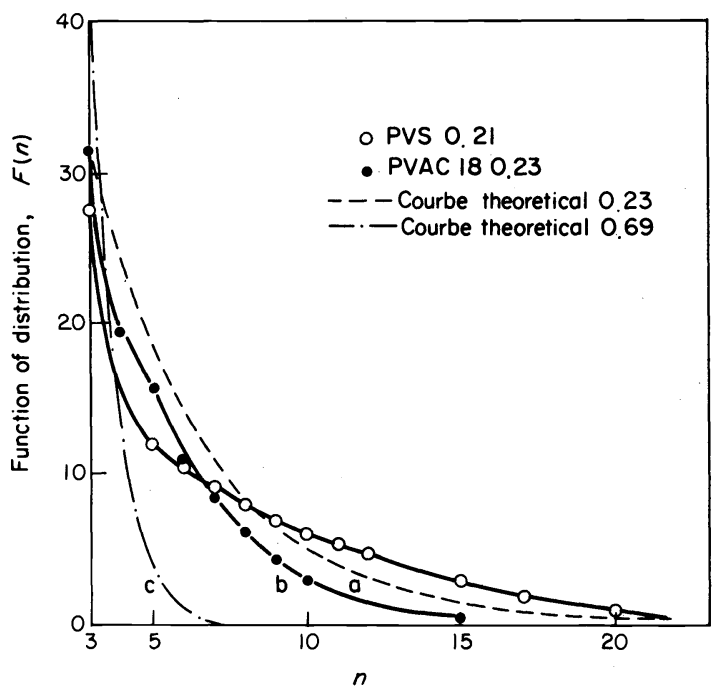

Fig. 10. Distribution of sequences of conjugated double bonds obtained after dehydration of partly sulfopropylated PVA. Numbers indicate the degree of substitution of the alcohol functions. Full lines are experimental curves; dotted lines are curves calculated under assumption of random grafting.

lation exhibits a sequential character. In other words, the reactivity of a PVA unit vicinal to a substituted one is enhanced.

Conversely, on Fig. 11, it can be seen that the distribution of double bonds in a PVA partly substituted by octadecyl chains and subsequently submitted to dehydration is below that curve calculated for the same DS under assumption of random placement of the grafts. This would rather indicate that the Williamson reaction when carried out on a polymer chain involves retardation.

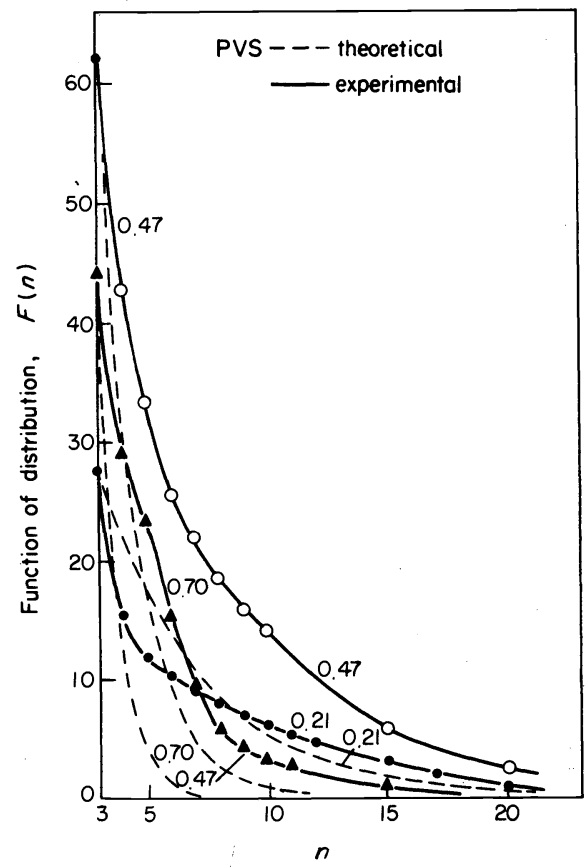

Fig. 11. Comparison of the distribution of sequences of conjugated double bonds obtained after dehydration for two partly substituted PVA: $O$ sulfopropylated to $21 \%$, alkylated with $\mathrm{C}_{18} \mathrm{H}_{37} \mathrm{Br}$ to $23 \%$, dotted line is calculated assuming random grafting for a DS of $23 \%$.
However X-ray diffraction experiments ${ }^{45}$ showed that the paraffinic side chains tend to crystallize in double layers, and the crystallization process itself was studied by differential scanning calorimetry, ${ }^{46}$ on PVA of various DS. As long as the DS is low, the melting point of the parafinic chains is somewhat decreased, but when more than $10 \%$ of the units have been substituted the melting point, the crystal structure, and the layer thickness does not change anymore with increasing DS. Furthermore the variation of the melting enthalpy vs the weight fraction of paraffinic chains in the polymer is linear, above $10 \%$ substitution.

It follows from this investigation that the reaction of alkylbromide onto the alcoholate of PVA is kinetically an auto-retarded reaction: the probability of reaction for an $-\mathrm{ONa}$ function vicinal to a substituted unit is decreased. But it is nevertheless in some way a sequential grafting process, because of the hydrophobic interaction between these long apolar paraffinic chains in a very polar reaction medium. From crystallographic and DSC data it follows that the average distance between two successive octadecyl chains along the chain is of the order of 3 units. It may be 2 or 4 , but to attain such regular double layers of crystallized side chains, even for low degrees of substitution, one has to assume that the grafting process exhibits a "sequential" character involving, say, one unit out of 3 .

This is an interesting case where the placement of substituted units is determined by intramolécular phase separation effects, rather than by purely chemical factors of reaction enhancement or retardation.

\section{CONCLUSION}

In this report our aim was to illustrate with recent examples taken from the activity of the CRM the trends of chemical work on modification of polymers. It is necessary, in each case, to accurately characterize the samples obtained, which are usually copolymers of transformed and of untouched units. This characterization should not involve solely chemical analysis or spectrographic determination, it should also include further techniques aimed to determine placement of substituted units (NMR, flash pyrolysis) and kinetic data whenever possible, in order to establish whether the enhancement or retardation effects are compatible with the distribution of untouched and of substituted units along the chain.

The case of multiple reactions is even more complicated, since it first requires chemical means to accurately determine at least 3 different types of units, and sometimes even more, as in the example shown. A quantitative description of the phenomena involved is not possible, in that case, but by careful comparison with model-molecules one can get information about the effect of neighboring units along the chain. Here also the more independent characterization techniques are used, the better it is for a satisfactory description (even if qualitative) of the phenomena involved.

Finally the third example quoted was meant to show that factors other than purely chemical (steric, ion interactions, dipole interactions) may influence the way a reaction proceeds. In the case which was examined the tendency of the paraffinic chains to crystallize and the hydrophobic interaction between them led to a sequential grafting process, even though the vicinal units of a substituted unit cannot (or can hardly) undergo the reaction of substitution. This means that whenever a 
reaction is carried out on a polymer chain, many factors may influence the reactivity of the individual units including some which do not appear at first sight as purely chemical ones.

Acknowledgements-The results reported here have been obtained by Dr. J. C. Galin and his coworkers, MM. J. J. Bourguignon and R. Roussel, and by Dr. Ph. Gramain together with Dr. Lemoigne. The author gratefully acknowledges their help in preparing this lecture.

\section{REFERENCES}

'E. Klesper, Chemical Reactions on Polymers. (J. A. Moore, ed.) Reidel, Dordrecht, p. 139(1973). (a) E. Klesper, A. Johnson and W. Gronski, Makromol. Chem. 160, 167 (1972).

${ }^{2}$ H.J.Harwood, Chemical Reactions on Polymers. (J.A. Moore,ed.) Reidel, Dordrecht, p. 188 (1973).

${ }^{3}$ E. A. Boucher, Trans. Faraday Soc. 68, 2281, 2293 (1972).

${ }^{4}$ N. Platé, Chemical Reactions of Polymers. (J. A. Moore, ed.), Reidel, Dordrecht, p. 169 (1973). (a) N. Platé, Europ. Polymer J. suppl. 517 (1969).

${ }^{5}$ E. Klesper et al., Makromol. Chem. 175, 523, 535 (1974).

${ }^{6}$ R. M. Fuoss, M. Watanabe and B. D. Coleman, J. Polymer Sci. 48, 5 (1960).

${ }^{7} \mathrm{~J}$. Morcelet-Sauvage and C. Loucheux, Makromol. Chem. 176, 315 (1975).

${ }^{8} \mathrm{~J}$. Moens and G. Smets, J. Polymer Sci. 23, 931 (1957).

${ }^{9}$ M. Higushi and R. Senju, Polymer J. 3, 370 (1972).

${ }^{10} \mathrm{H}$. Morawetz, Chemical Reactions of Polymers. (E. M. Fettes, ed.) Interscience, New York. p. 10 (1964).

${ }^{11}$ J. P. Roth, P. Rempp and J. Parrod, J. Polymer Sci. 64, 1347 (1964).

${ }^{12}$ M. L. Bender and M. C. Neveu, J. Am. Chem. Soc. 80, 5388 (1958).

${ }^{13}$ N. A. Platé, A. D. Limanovich, O. V. Noa and V. I. Golyakov, Polymer Sci. USSR. 11, 2511 (1969).

${ }^{14}$ O. V. Noa, A. L. Toom, N. V. Vasil'ev, A. D. Limanovich and N. A. Platé, Polymer Sci. USSR. 15, 986 (1973).

${ }^{15}$ N. A. Platé et al., J. Polymer Sci. (Chem.) 12, 2165 (1974).

${ }^{16}$ N. W. Johnston and H. J. Harwood, J. Polymer Sci. C-22, 591 (1969). Macromolecules 2, 221 (1969). Macromolecules 3, 566 (1970).

${ }^{17} \mathrm{G}$. Smets, Chemical Reaction of Polymers. (E. M. Fettes, ed.) Interscience, New York, p. 79 (1964).
${ }^{18} \mathrm{G}$. Van Beylen, Stereochemistry of Macromolecules. (A. D. Ketley, ed.), Dekker, New York. p. 333 (1968).

${ }^{19} \mathrm{H}$. Moravetz, Macromolecules in Solution. Interscience, New York, p. 409 (1965)

${ }^{20}$ N. W. Johnston, Macromolecules 5, 87 (1972).

${ }^{21}$ P. J. Flory, J. Am. Chem. Soc. 61, 1518 (1939).

${ }^{22}$ H. Abe, K. Imai and M. Matsumoto, J. Polymer Sci. B-3, 1053 (1965).

${ }^{23}$ G. Schroeder, Makromol. Chem. 96, 227 (1966).

${ }^{24}$ H. L. Cohen, D. G. Borden and L. M. Minsk, J. Org. Chem. 26, 1274 (1961).

${ }^{25}$ E. J. Corey and D. Seebach, J. Org. Chem. 31, 4097 (1966).

${ }^{26}$ R. L. Sowerby and R. M. Coates, J. Am. Chem. Soc. 94, 4758 (1972).

${ }^{27}$ E. J. Corey and M. Chaykovsky, J. Am. Chem. Soc. 86, 1639 (1964). 87, 1345 (1965).

${ }^{28}$ G. A. Russel, E. T. Sabourin and G. Hamprecht, J. Org. Chem. 34, 2339 (1969).

${ }^{29}$ E. J. Corey and T. Durst, J. Am. Chem. Soc. 90, 5548 (1968).

${ }^{30}$ N. N. Goldberg, L. B. Barkley and R. Levine, J. Am. Chem. Soc. 73, 430 (1951).

${ }^{31}$ F. Arranz and J. C. Galin, Makromol. Chem. 152, 185 (1972).

${ }^{32}$ F. C. Baines and J. C. Bevington, J. Polymer Sci. A-1, (6) 2433 (1968).

${ }^{33}$ A. D. Limanovich and V. Ya Shtern, J. Polymer Sci. C-16, 1375 (1967).

${ }^{34}$ S. Teramachi and Y. Kato, J. Macromol. Sci. A-4, 1785 (1970).

${ }^{35}$ S. Teramachi and Y. Kato, Macromolecules, 4, 54 (1971).

${ }^{36} \mathrm{~W}$. Bushuk and H. Benoit, Can. J. Chem. 36, 1616 (1958)

${ }^{37} \mathrm{H}$. Benoit and D. Froelich, Light Scattering from Polymer solutions.(M. Huglin,ed.) Acad.Press, London. p.467 (1972).

${ }^{38} \mathrm{~J}$. C. Jungers and L. Sajus, Analyse Cinétique de la Transformation Chimique. (Tome I, ed.) Techn. Paris. p. 413 (1967).

${ }^{39} \mathrm{~F}$. Dolle, J. Le Moigne and P. Gramain, Europ. Polymer J. 6, 1227 (1970).

${ }^{40}$ J. C. Galin, J. Appl. Polymer Sci. 15, 213 (1971).

${ }^{41}$ E. J. Goethals and G. Natus, Makromol. Chem. 116, 152 (1968).

${ }^{42} \mathrm{~J}$. Le Moigne and P. Gramain, Europ. Polymer J. 8, 703 (1972).

${ }^{43}$ J. Le Moigne and P. Gramain, J. Appl. Polymer Sci. 17, 3555 (1973).

${ }^{44}$ Y. K. Kirilenko, A. I. Meos and L. A. Vol'f, Zh. Prikl. Khim. 38, 2991 (1965).

${ }^{45}$ A. Mathis, J. Le Moigne and P. Gramain, Europ. Polymer J. 9 , 283 (1973).

${ }^{46} \mathrm{~J}$. Le Moigne and P. Gramain, Europ. Polymer J. 10, 401 (1974). 